\title{
UM EXEMPLO DE PROJETO INTEGRADOR: PROJETO DE UM BRAÇO ROBÓTICO DE CINCO EIXOS NO CURSO DE MANUFATURA AVANÇADA DO CENTRO PAULA SOUZA
}

DOI: $10.37702 / 2175-957 X . C O B E N G E .2021 .3387$

Arthur Vieira Netto Jr - arthur.netto01@fatec.sp.gov.br

Centro Paula Souza

Rua fioravante sajo 133

18087-012 - Sorocaba - SP

Samuel Mendes Franco - samuelfranco@fatecsorocba.edu.br

Centro Paula Souza

Av. Eng. Carlos Reinaldo Mendes, 20152015

18013-280 - Sorocaba - SP

Edemir Celso Mantovani - edemir.mantovani@fatec.sp.gov.br

Centro Paula Souza

Rua Dom Pedro II 100

18087-012 - Sorocaba - SP

Resumo: $O$ presente trabalho refere-se à implementação do conceito de aprendizagem baseada em projetos (ABProj) no curso de tecnologia de Manufatura Avançada do Centro Paula Souza. O conceito de um projeto integrador foi materializado por meio do desenvolvimento e fabricação de um braço robótico de cinco eixos. Esse robô revelou-se uma boa ferramenta pedagógica, em decorrência de sua aplicabilidade teórica e prática, com possibilidade de introdução de modificações no projeto, ampliando o leque de aplicação no ensino e pesquisa. Para a implementação do conceito ABProj no contexto e realidade do curso de Manufatura Avançada foram necessárias adaptações e envolvimento de alunos e professores, bem como adequação de infraestrutura dos laboratórios e salas de aula. Depois de um período inicial de adaptação e assimilação do novo método, houve maior integração interdisciplinar facilitando a transmissão dos conhecimentos e aumentando a confiança em relação às vantagens da aprendizagem baseada em projetos.

Palavras-chave: Metodologia ativa. Robótica. Projeto Integrador. Mecatrônica. 


\section{INTRODUÇÃO}

O uso de metodologias novas de ensino tem se apresentado como uma opção importante no contexto dos cursos de engenharia e tecnologia. Em face da rápida evolução da tecnologia dentro da Indústria 4.0, as instituições de ensino superior têm criado novos cursos de tecnologia para suprir o mercado com profissionais qualificados nesse novo contexto tecnológico. O Curso de Tecnologia em Manufatura Avançada foi criado com esse intuito, pelo Centro Paula Souza, para ser ministrado pelas suas Faculdades de Tecnologia, abrangendo várias cidades por todo o Estado de São Paulo.

A Faculdade de Tecnologia de Sorocaba (FATEC-SO) iniciou esse curso em 2018 tendo como base da sua estrutura pedagógica o conceito do Projeto Integrador (PI). Segundo Cavali (2017) o Projeto Integrador permite ao aluno ser participante da sua própria construção do conhecimento por meio da investigação e pesquisa sobre uma determinada área do conhecimento. A Robótica, que propõe o desenvolvimento de vários tipos de robôs foi escolhida como tema principal do Projeto Integrador, pois agrega vários conceitos do conhecimento o que possibilita a integração de várias disciplinas que são ministradas no referido curso.

Para servir como um modelo físico real de um robô, adequado ao conceito de Projeto Integrador foi desenvolvido e construído um braço robótico de cinco eixos, baseado em um projeto "open source" no qual a estrutura desse robô é fabricada em plástico ABS por meio de manufatura aditiva (impressão 3D), sendo acionado por seis motores de passo. O controle desses motores é realizado por uma plataforma Arduíno ligada a uma placa Ramps 1.4, que efetua a interface entre sensores, drivers e displays acoplados ao robô.

O braço robótico serve então como elo principal de ligação com outras disciplinas dentro do projeto pedagógico como, por exemplo, a disciplina de Lógica de Programação em Arduíno; a disciplina de Desenho Técnico, a disciplina de Tecnologia Mecânica, a disciplina de Eletrônica, a disciplina de Matemática, entre outras. Esse fato propicia ao aluno uma interação real com os conteúdos das disciplinas, pois o conhecimento que está sendo adquirido pode ser visto e testado por meio do Projeto Integrador, transformando os conceitos teóricos em ações práticas sedimentando a transferência do conhecimento de modo criativo e eficiente.

Outro ponto importante é o desafio que pode ser colocado para os alunos no sentido de reprojetar o braço robótico para, por exemplo, conseguir carregar mais carga, ou ser mais veloz, ou aumentar a precisão de posicionamento espacial. Segundo Moura (2018), a aprendizagem baseada em projetos é um modelo que se destaca por envolver os alunos e incentivá-los a pesquisar problemas práticos e relevantes para o seu aprendizado sendo, portanto, ideal para cursos que pretendem atingir um bom nível na relação ensino e aprendizagem.

\section{O PROJETO DEDAGÓGICO DO CURSO DE MANUFATURA AVANÇADA}

Com o intuito de aproveitar da melhor forma as oportunidades proporcionadas pela tecnologia no ensino tecnológico e ao mesmo tempo mover-se em direção à inovação, sustentabilidade e emprego, o Centro Paula Souza elaborou um Curso Superior de 
Tecnologia em Manufatura Avançada. Este Curso tem como objetivo formar tecnólogos qualificados para enfrentar os desafios da quarta revolução industrial.

Abordando conceitos que vão desde a concepção do produto, projeto, desenvolvimento do processo de Manufatura, integração entre processos e máquinas, assim como agregando as tecnologias voltadas para a Indústria 4.0 como Sistemas Automatizados, Simulação Computacional, Realidade Aumentada e Virtual, Sistemas Integrados, Robótica Autônoma, Internet das Coisas, Big Data, Segurança da Informação e Manufatura Aditiva.

Para promover a formação dos alunos dos cursos de tecnologia, os professores têm procurado utilizar novas metodologias de ensino e aprendizagem que facilitem o desenvolvimento das competências (habilidades, conhecimento e atitudes), que são essenciais para a vida profissional desse mesmo aluno.

Uma dessas metodologias de ensino é a Aprendizagem Baseada em Projetos (ABProj). Segundo Gonçalves (2020), a Aprendizagem Baseada em Projetos é uma metodologia em que os alunos são envolvidos com tarefas e desafios com o objetivo de desenvolver um produto ou um projeto.

Para formar profissionais engajados no processo de aprendizagem contínua ao longo da vida, criativos, preparados para a solução de problemas na área da Manufatura Avançada e da Indústria 4.0, serão utilizadas as metodologias ativas em seus componentes curriculares e Projetos Integradores.

\subsection{Metodologia do Projeto Integrador}

A dinâmica das aulas seguiu o modelo de aprendizado por competências, onde o aluno é estimulado a buscar as respostas aos questionamentos apresentados em sala/laboratório. A classe foi dividida em cinco grupos de oito alunos. Cada grupo recebeu como tarefa a desmontagem virtual, a identificação dos componentes, o conhecimento dos componentes, o redesenho dos componentes, a fabricação de um novo componente e a montagem do braço robótico, baseado no modelo apresentado e com os aperfeiçoamentos que poderão ser incorporados. A tabela 1 apresenta uma lista de objetivos de aprendizagem que 0 aluno deve atingir.

Tabela 1 - O que tem que ser conhecido sobre um Robô.

Como funciona uma máquina?
Como o robô funciona?
Como ocorrem os movimentos?
Como é o sistema mecânico?
Como é o sistema eletrônico?
Como funcionam os movimentos controlados?
Como programar os controladores?
Quais são os conhecimentos de cinemática?
Quais são os conhecimentos dos elementos de máquinas?
Quais são os conhecimentos de desenho?
Quais são os conhecimentos sobre os processos de fabricação?
Fonte: Autores.

As sequências de trabalho dos alunos foram divididas em duas partes, conforme mostra a tabela 2, à esquerda refere-se à análise do produto existente e a direita trata o produto a ser construído. 
Tabela 2 - Trabalho a ser realizado pelos alunos no primeiro semestre.

\begin{tabular}{|c|l|c|}
\hline & \multicolumn{1}{|c|}{ Robô - Modelo } & Robô - Confecção e Construção \\
\hline \multirow{3}{*}{ Etapas } & Análise & Planejamento \\
\cline { 2 - 3 } & Desmontagem & Fabricação \\
\cline { 2 - 3 } & Montagem & Montagem \\
\hline
\end{tabular}

Fonte: Autores.

Os grupos realizaram uma série de análises dos componentes, visando identificar, conhecer, tomar contato com os elementos, conhecer as normas que regem os mesmos, ensaios necessários e os tipos de materiais.

$\mathrm{Na}$ análise da parte mecânica, o aluno deveria identificar e compreender os elementos de máquinas, a precisão, o volume de trabalho e a carga de trabalho. Em relação aos componentes eletrônicos o aluno deveria identificar e compreender 0 funcionamento dos seguintes itens: motor de passo, servo motor, drive, chave fim de curso, sensores, atuadores e a linguagem de programação para Arduino.

No plano de desmontagem do braço robótico o aluno deveria: analisar o braço robótico, elaborar instruções de desmontagem, analisar a fixação e o procedimento para retirar dos elementos de máquinas e os componentes eletrônicos, catalogar os componentes, medir as peças e desenhar as peças.

Após confecção dos desenhos do braço robótico em estudo, seria iniciada a fabricação de um novo modelo, imprimindo as peças utilizando a manufatura aditiva; neste instante, as discussões sobre modificações que promovam melhorias foram de grande importância no aprendizado entre os diversos grupos de trabalho para colocar em prática os ensinamentos adquiridos.

A montagem das partes mecânica, elétrica e a programação do braço robótico, seria composta pela elaboração do plano de montagem, análise dos acoplamentos, torque dos elementos de fixação, ajustes dimensionais e testes; além da análise dos resultados. robótico.

$\mathrm{Na}$ sequencia serão apresentados em detalhes o projeto e a construção do braço

\section{PROJETO E CONSTRUÇÃO DO BRAÇO ROBÓTICO DE CINCO EIXOS}

O motivo da construção do braço robótico foi estabelecer um modelo real para o Projeto Integrador que estivesse dentro do contexto tecnológico nacional e mundial. Ao focalizar esse contexto, a Quarta Revolução Industrial (Indústria 4.0), conceito recente que tem mudado fundamentalmente a sociedade e economia mundiais nos últimos anos, nota-se a crescente importância da Robótica como fator estratégico para o avanço tecnológico.

Segundo Lorenz et al. (2015), a Industria 4.0 está alicerçada em nove pilares do avanço tecnológico, sendo um deles a Robótica. Um tipo de robô globalmente representativo para a Indústria 4.0 é o braço robótico articulado de cinco/seis eixos rotacionais $(5 R / 6 R)$ que foi a escolha mais adequada para ser utilizado no Projeto Integrador.

\subsection{Desenvolvimento do braço robótico.}

O projeto é baseado no modelo oriundo da Universidade da Catalunha sendo aberto na sua concepção (open source) e permitindo alterações na sua estrutura (BCN3D, 
2020). Trata-se de um robô de cinco eixos que pode ser produzido, modificado e ampliado para ser aplicado em pesquisa e educação.

Um dos problemas encontrados pelo Departamento de Engenharia da Universidade da Catalunha era o alto custo dos materiais que os alunos de graduação deveriam utilizar para seus estágios. Portanto, um braço robótico de código aberto, modificável pelos alunos e reproduzível a baixo custo, poderia cobrir variadas fases de treinamento existentes: projeto mecânico, automação, programação industrial, entre outras (BCN3D, 2020).

O mesmo problema foi encontrado no curso de Manufatura Avançada do Centro Paula Souza, de modo que esse modelo de robô poderia contribuir em muito para aperfeiçoar o processo de formação tecnológica dos atuais alunos. Uma vez definido o projeto inicial foi feita uma parceria com a empresa Flexbras residente no Parque Tecnológico de Sorocaba, que produz impressoras com a tecnologia de manufatura aditiva, para a fabricação dos componentes do braço robótico.

\subsection{Estrutura, mecânica e eletrônica de acionamento do braço.}

Todo o projeto original do braço robótico foi analisado e algumas modificações foram realizadas para melhorar a resistência estrutural do robô, bem como a rigidez do conjunto entre os eixos rotacionais, os motores e as correias. Toda a estrutura do robô foi feita em peças que foram impressas (manufatura aditiva) em plástico ABS (Acrilonitrila butadieno estireno). Esse plástico foi escolhido porque suporta temperaturas de até $110^{\circ} \mathrm{C}$ sem apresentar deformação mecânica, visto que os motores de passo podem atingir a temperatura de $80^{\circ} \mathrm{C}$ normalmente durante o seu funcionamento (VIEIRA, 2019).

O robô construído utiliza na sua eletrônica de acionamento e controle duas placas Arduíno Mega 2560, uma placa Ramps 1.4, e na sua eletrônica de potência, seis drivers TB6600 para acionamento dos motores de passo. Possui seis motores de passo ao todo; dois modelos NEMA 23; três NEMA 17 e um NEMA 14 além de um servo motor para movimentação da garra, ver figura 1.

Figura 1 - Componentes do sistema eletrônico de controle e potência.

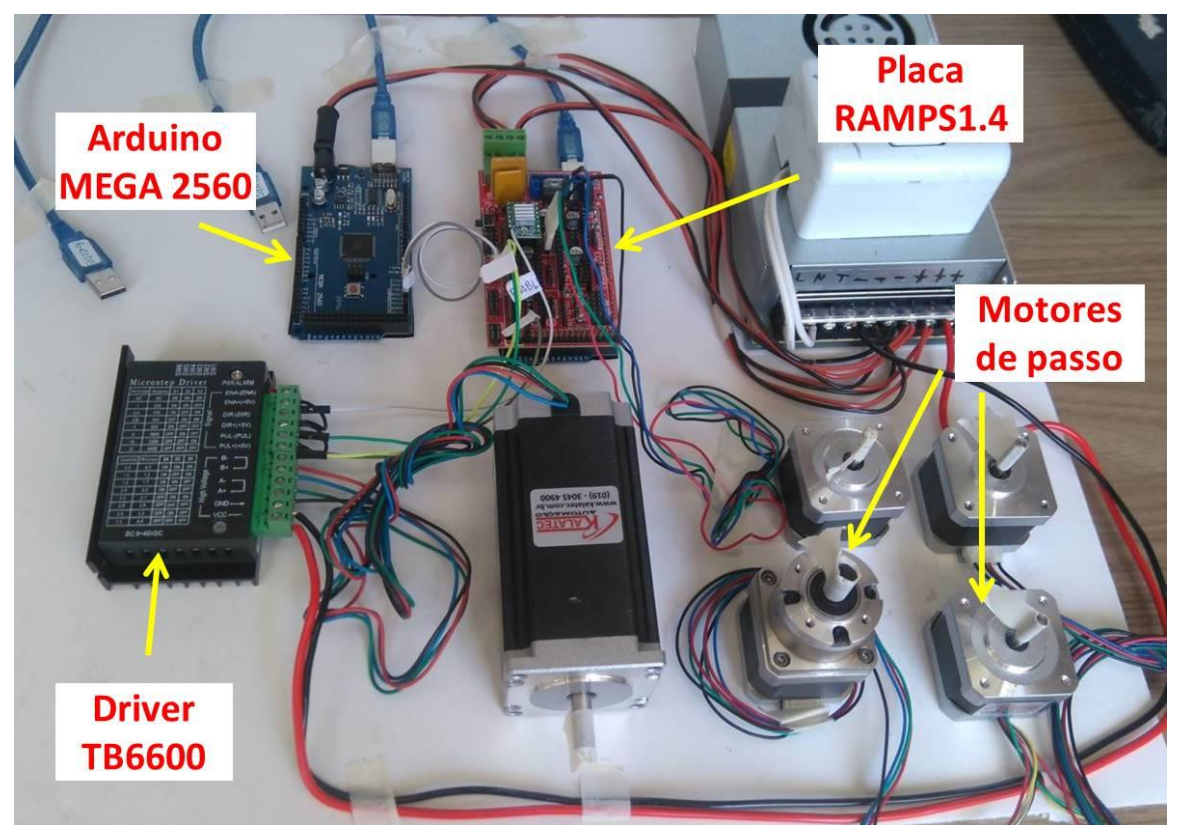

Fonte: Autores. 
Todos os eixos rotacionais são dotados de rolamento de esferas (608ZZ, 625ZZ, $624 Z Z, 623 Z Z$ ) e o acionamento mecânico é realizado por conjuntos de polias dentadas T5 e correias dentadas T5 de largura $15 \mathrm{~mm}$. Na figura 2 abaixo são apresentados alguns detalhes construtivos do robô em relação ao acionamento dos eixos rotacionais.

Figura 2 - Vista mostrando o posicionamento dos motores no segundo eixo rotacional.
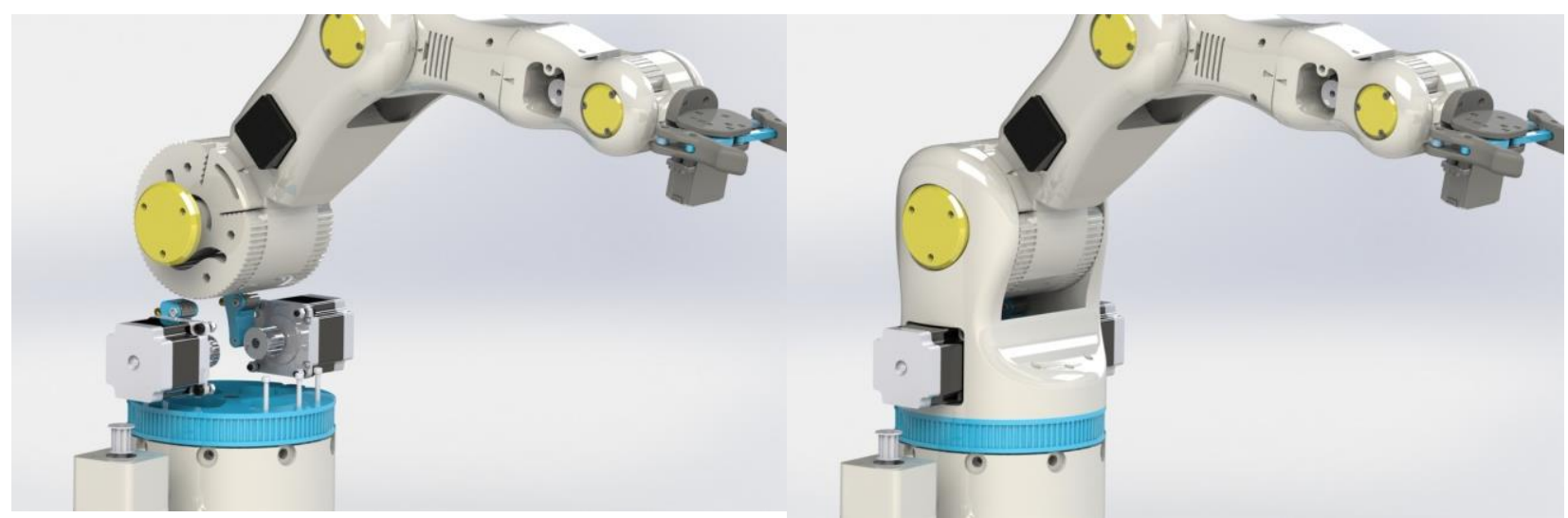

Fonte: Autores.

Esse robô pode ser programado em código G (G-code) para realizar movimentos complexos com acionamento simultâneo de quatro eixos (Marlin, 2020). A velocidade angular e aceleração angular de cada eixo rotacional podem ser definidas dentro de uma larga faixa de atuação, desde movimentos com pouca aceleração e velocidade até com máxima aceleração e alta velocidade.

O braço robótico tem capacidade de mover e carregar peças e ferramentas com até $1 \mathrm{~kg}$. Seu raio de ação para movimentação de carga é de $700 \mathrm{~mm}$ em $340^{\circ}$.

Podem ser programados até 10 movimentos e gravados na placa do Arduíno para posterior uso. Abaixo, na figura 3 , é mostrado o braço robótico completo em movimentação em teste de desempenho de velocidade de trabalho e repetibilidade.

Figura 3 - Braço robótico em teste de velocidade de trabalho e repetibilidade.

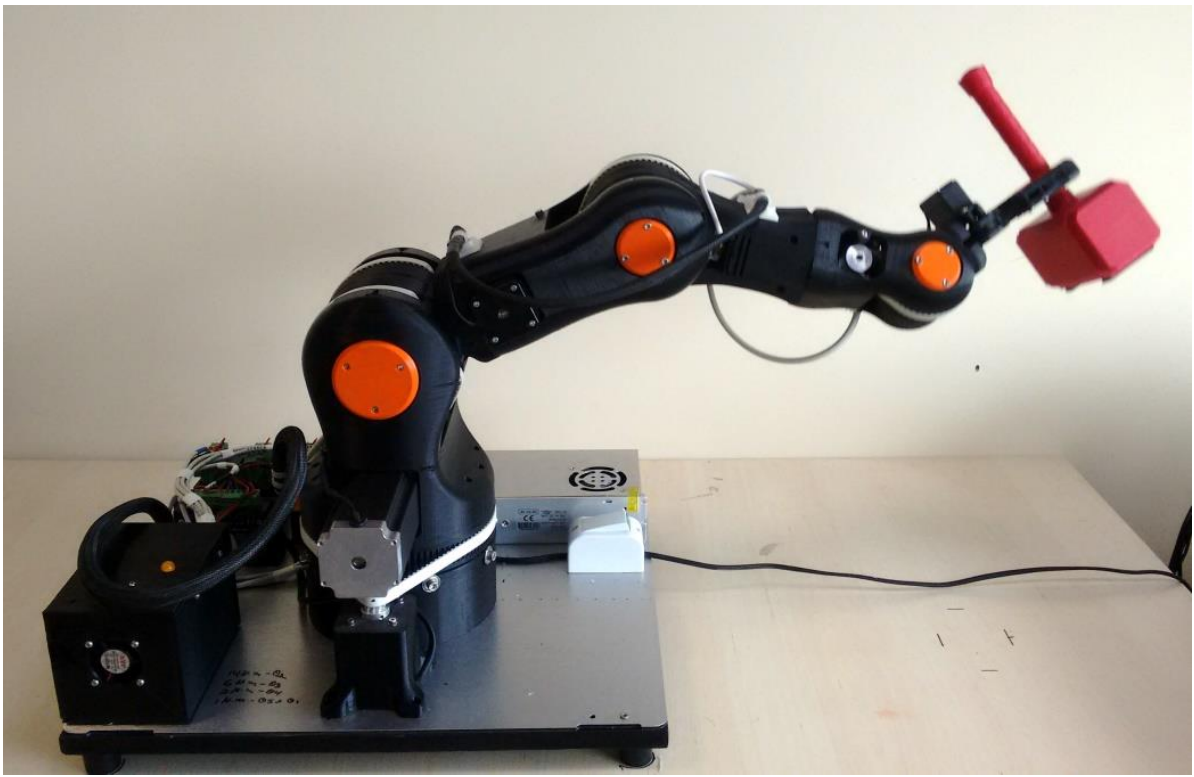

Fonte: Autores. 


\section{$4 \quad$ RESULTADOS}

A implantação do projeto pedagógico no curso de Manufatura Avançada utilizando o conceito de aprendizagem baseada em projetos passou por algumas fases de adaptação do novo método de ensino. Essas fases podem ser resumidas em adaptação dos alunos, adaptação dos professores e adaptação dos laboratórios e salas.

Em relação à atuação docente, houve a necessidade de que o conteúdo abordado fosse apresentado de forma clara e relacionado ao projeto do robô, moldando as disciplinas para que atendessem o projeto integrador dentro da grade curricular já determinada. Para tanto, várias reuniões de planejamento docente foram necessárias, para definição de um objetivo comum a ser atingido no aprendizado discente.

Tomando como exemplo apenas as disciplinas do primeiro semestre, o arranjo do conteúdo programático destas procurava suprir as necessidades dos alunos frente ao projeto integrador, que também foi modelado como uma disciplina a mais no contexto pedagógico, denominada de Desenvolvimento Integrado do Produto, a qual pode ser vista na figura 4.

Figura 4 - Relação das disciplinas do $1^{\circ}$ semestre em função do projeto integrador.

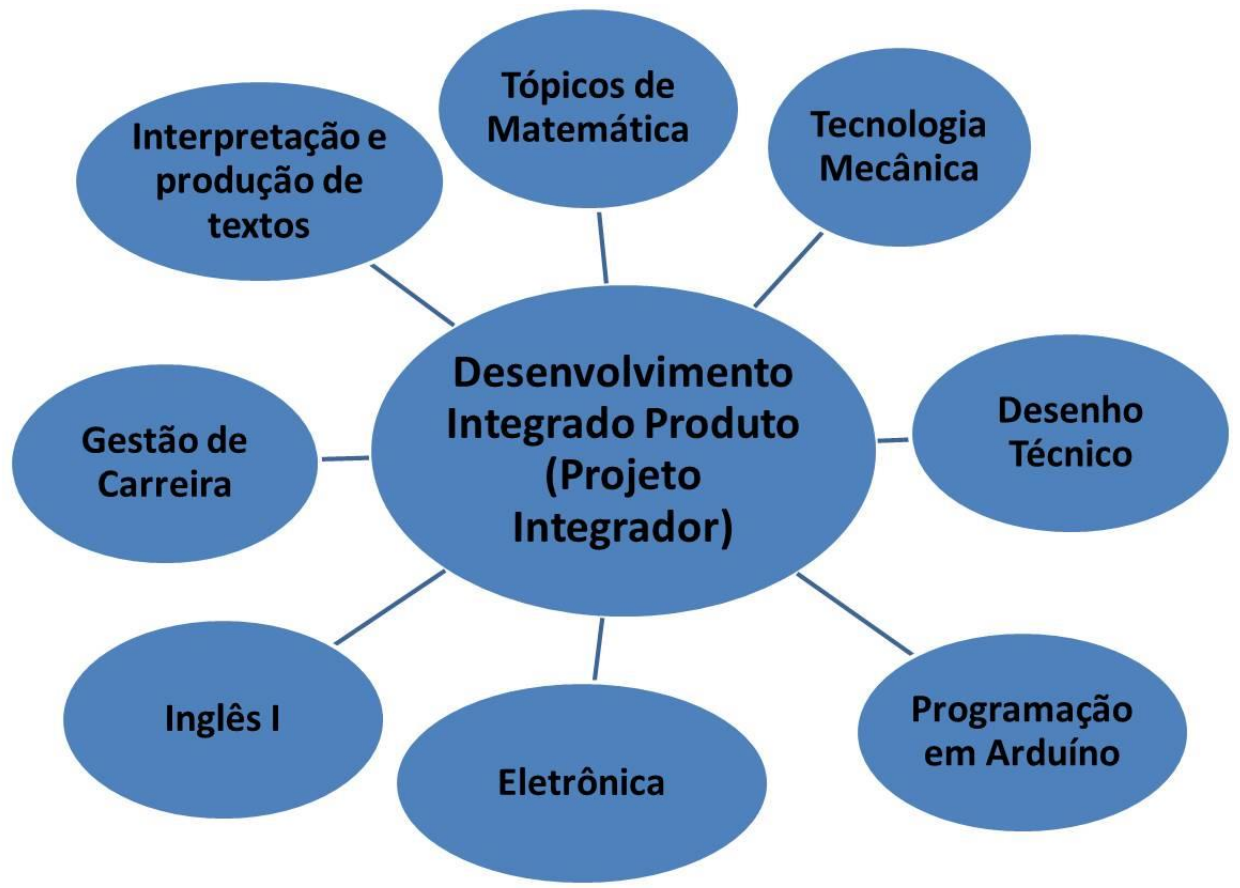

Fonte: Autores.

Essa estrutura de integração entre as disciplinas permitiu que os conhecimentos teóricos e práticos fossem transferidos aos alunos de modo mais coordenado e construtivo, pois todos os conteúdos tinham como referência inicial o projeto do braço robótico.

Em relação aos alunos a adaptação à nova metodologia foi mais lenta, pois a maioria dos ingressantes no curso vinham de escola pública que sempre adotou o método tradicional de exposição teórica dos conteúdos programáticos. A transição para uma sala de aula inovadora na qual a pró-atividade do aluno era exigida a todo o momento foi aos poucos sendo compreendida. No início do curso, a maioria dos alunos ainda se 
comportava de modo passivo em relação às aulas, refletindo o comportamento da escola tradicional da qual eram provenientes.

Outro sintoma dessa adaptação inicialmente lenta foi o fato de que no método novo a avaliação do aluno não era feita somente por meio de provas teóricas sendo balizadas por notas (Avaliação Somativa), mas também por meio da própria interatividade do aluno com a sua equipe, com as aulas e com o projeto integrador (Avaliação Formativa). Ao longo do semestre a assimilação do novo método foi sendo mais bem compreendida pelos alunos facilitando a transmissão dos conhecimentos e aumentando a confiança em relação às vantagens da aprendizagem baseada em projetos.

Com relação à adaptação de salas de aula e laboratórios, pode-se dizer que a eficiência em relação à compreensão dos vários conhecimentos que o aluno deve ter é fortemente dependente de como os laboratórios estão equipados com ferramentas, máquinas para prototipagem, softwares para desenho mecânico, para programação de placas eletrônicas e instrumentos eletrônicos. A construção do projeto integrador passa necessariamente pela fabricação e montagem de um conjunto de peças, motores, fiação e acoplamentos que só pode ser feito com maquinário e instrumental próprios.

Em resumo as adaptações necessárias à nova metodologia foram realizadas de modo que o curso de Manufatura Avançada realmente adotasse uma postura didática proativa em relação aos alunos e mais colaborativa com os professores.

\section{CONSIDERAÇÕES FINAIS.}

As aplicações das novas metodologias de ensino nos cursos de tecnologia devem passar necessariamente por um processo de compartilhamento de experiências entre professores, alunos e principalmente gestores, formando uma rede de informação, a qual pode validar ou não as adaptações ao modelo pedagógico. Isso leva a crer que uma mudança cultural e educacional deva ocorrer paulatinamente ao longo dos próximos anos nessas instituições de ensino, em função das urgentes demandas por profissionais atualizados e com competência em suas áreas de atuação, com maior capacitação para o trabalho em equipe e busca pela informação.

Desse modo a experiência relatada aqui poderá ser útil para se tenha uma maior eficácia na relação entre a oferta de conhecimentos e a devida absorção e compreensão dos mesmos pelos alunos.

\section{REFERÊNCIAS}

BCN3D; BCN3D MOVEO - A fully Open Source 3D printed robot arm. Disponível em: https://www.bcn3dtechnologies.com/en/bcn3d-moveo-the-future-of-learning/ . Acesso em: 21/10/2020.

CAVALI, RUBIA C.; Metodologias Ativas no Ensino Superior - Uma Experiência com Projetos Integradores. In: EDUCERE - XIII CONGRESSO NACIONAL DE EDUCAÇÃO PUCPR, 2017, Curitiba. Anais... Curitiba: PUCPR, 2017. p. 20565-20575. Disponível em: https://educere.bruc.com.br/arquivo/pdf2017/26661 13551.pdf. Acesso em 18/03/2021.

GONÇALVES, A. M.; A prática docente e a utilização de metodologias ativas na educação técnica profissional. Dissertação de Mestrado em Gestão do Desenvolvimento Educacional Profissional. Centro Paula Souza, p.100, São Paulo, 2020. 
LORENZ, M.; RUBMANN, M.; GERBERT, P.; WALDNER, M.; JUSTUS, J.; ENGEL. P.; HARNISCH, M.; Industry 4.0: The Future of Productivity and Growth in Manufacturing Industries. Boston Consulting Group (BCG), New York, 2015.

MARLIN; G-Code Index. Disponível em: https://marlinfw.org/meta/gcode/ Acesso em: 25/10/2020.

MOURA, EUNICE E.; Projeto Integrador como instrumento de pesquisa no processo de desenvolvimento do aprendizado. In: I SEMINÁRIO EM PESQUISA ACADÊMICA E CIENTÍFICA NA PRÁTICA DOCENTE, 2018, Curitiba. Anais... Curitiba: Faculdade Bagozzi, 2018, p.169. Disponível em: https://faculdadebagozzi.edu.br/wpcontent/uploads/2018/08/ANAIS-Semin\%C3\%A1rio-em-Pesquisa-Acad\%C3\%AAmica-eCient\%C3\%ADfica-na-Pr\%C3\%A1tica-Docente-BAGOZZI-25-e-26-de-julho-de-2018.pdf Acesso em 15/03/2021.

VIEIRA, A. N. J.; Construção de um Braço Robótico de Cinco Eixos, para Uso, Pesquisa e Ensino de Robótica Industrial. IX Workshop de Integração de Tecnologias e Aplicações na Indústria 4.0; PTS - Parque Tecnológico de Sorocaba, Sorocaba, outubro, 2019.

\title{
AN EXAMPLE OF AN INTEGRATIVE PROJECT: DESIGN OF A FIVE AXIS ROBOTIC ARM IN THE ADVANCED MANUFACTURING COURSE OF THE CENTRO PAULA SOUZA.
}

\begin{abstract}
This paper documents the introduction of project-based learning (ABProj) in the Advanced Manufacturing technology course at Centro Paula Souza, as well as its results, through the development and manufacture of a five-axis robotic arm as an integrative project. The robot proved to be a good pedagogical tool, due to its theoretical and practical applicability. Furthermore, it opened the possibility of project customization, expanding the range of applications for teaching and research. The success of the ABProj concept's implementation required the following adjustments in the context and reality of the Advanced Manufacturing course: (i) on-hand involvement of students and teachers; (ii) change in the infrastructure of laboratories and classrooms. By the end of this initial adaptation period, marked by the assimilation of the new method by students and teachers, the course experienced greater interdisciplinary integration, facilitating the transfer of knowledge and, thus, corroborating the argument of the advantages offered by project-based learning.
\end{abstract}

Keywords: Active methodology. Robotics. Integrative Project. Mechatronics. 